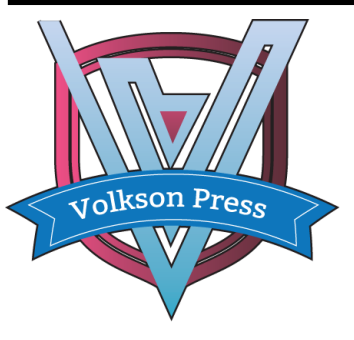

Contents List available at VOLKSON PRESS

New Materials and Intelligent Manufacturing (NMIM) DOI : http://doi.org/10.26480/icnmim.01.2018.130.132

Journal Homepage: https://topicsonchemeng.org.my/

ISBN: 978-1-948012-12-6

\title{
RESEARCH ON SELF BALANCING VEHICLE CONTROL BASED ON KINECT HUM AN POSTURE RECOGNITION
}

\author{
Jiayi Li*, Min Yan, Jianjun Zhu \\ Department of Automation, Jilin Institute of Chemical Technology Chengde Street, Jilin, China. \\ *Corresponding Author Email: washy59@sina.com
}

This is an open access article distributed under the Creative Commons Attribution License, which permits unrestricted use, distribution, and reproduction in any medium, provided the original work is properly cited

\section{ARTICLE DETAILS}

Article History:

Received 26 June 2018

Accepted 2 July 2018

Available online 1 August 2018

\section{ABSTRACT}

\begin{abstract}
The design of two wheeled self balancing vehicle was based on human posture recognition. The PC was used to obtain the human bone tracking data through Kinect camera and displayed it on the screen. By recognizing human movements or gesture, the result was concerted to control command and transmitted to the self balancing vehicle through Bluetooth, so that the vehicle can execute the movement of advancing, retreating and turning. The combination of human posture recognition technology and two wheeled self balancing vehicle can meet the needs of human-computer interaction control in complex environment and has a good reference value for the field of robot for surveying missions in complex environment.
\end{abstract}

\section{KEYWORDS}

Kinect, human posture recognition, self balancing vehicle, human-computer interaction

\section{INTRODUCTION}

With the continuous development of technology, human-machine interaction technology has gradually changed from traditional touch control of keyboard and mouse to somatosensory interaction mode based on action recognition $[1,2]$. The research significance of action recognition is that it can make the computer "understand" the various actions of the person directly, that is, it can identify the person's body movement language, and convert it into control instruction according to the predetermined rules to control the object [3]. The representative equipment of this new model is the Kinect 3D somatosensory photographer. The Kinect 3D somatosensory camera is a somatosensory peripherals of Microsoft [4]. It can be used to adjust the angle of the camera and can be operated directly through physical action or voice, so that it has a better field of view $[5,6]$.

This research is based on the new imaging principle of depth of field of Prime Sense and obtains the skeleton coordinate information through region eigenvalues of depth image and random forest algorithm. After matching the body movements with the control commands, the commands for the self balancing vehicle are transmitted by Bluetooth communication. The combination of body recognition technology and self balancing vehicle has a good reference value for robot exploration in complex environment.

\section{INTEGRATED SYSTEM DESIGN}

The system uses the Kinect 3D camera as the image acquisition module. The data between the PC and the self balancing vehicle is transmitted by Bluetooth, which sets the IP in the same section to establish the TCP connection and completes the two-way transmission of the data packet. The self balancing vehicle sends debugging information to the PC to confirm the car posture. The development platform is Visual Studio 2012 Kinect SDK. In this version, $\mathrm{C}++$ or $\mathrm{C} \#$ can be selected as development language, and color image and skeleton model data can be acquired through deep sensor. The controlled object is a two-wheeled selfbalancing vehicle. The vehicle is composed of STM32F103 MCU main control module, attitude sensor module, encoder, Bluetooth module, display module, motor drive module and voltage stabilization module. The attitude sensor MPU6050 can get the posture of the vehicle. The attitude sensor MPU6050 can get the posture of the vehicle. After the data is processed by MPU6050, the attitude of the vehicle is converted to the numerical value and sent to the microcontroller STM32F103. After comparing the actions recognized by Kinect with the action set, the self balancing vehicle performs the corresponding actions. Block diagram of the system is as shown in Figure 1.

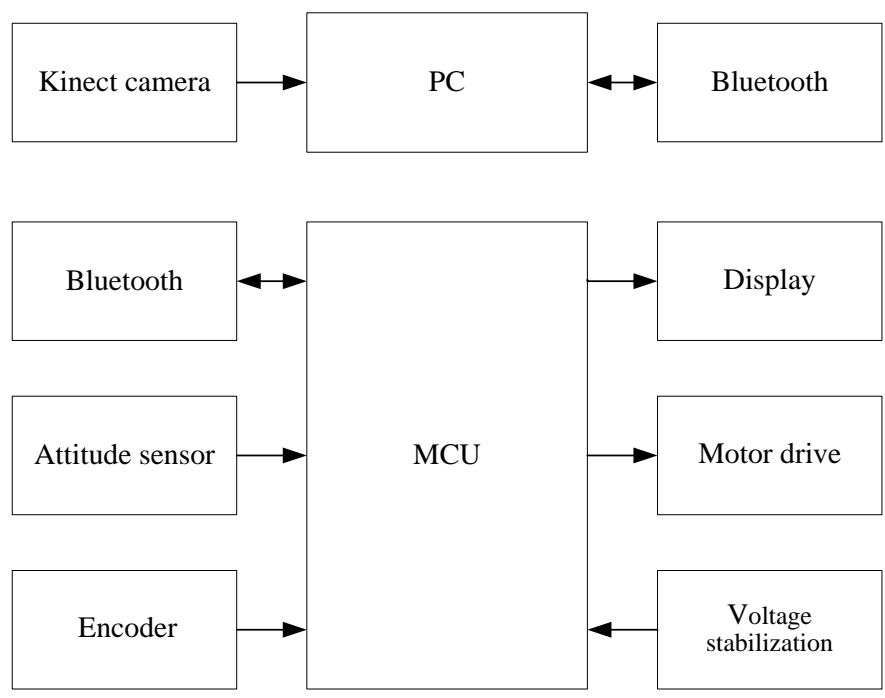

Figure 1: Block diagram of the system 


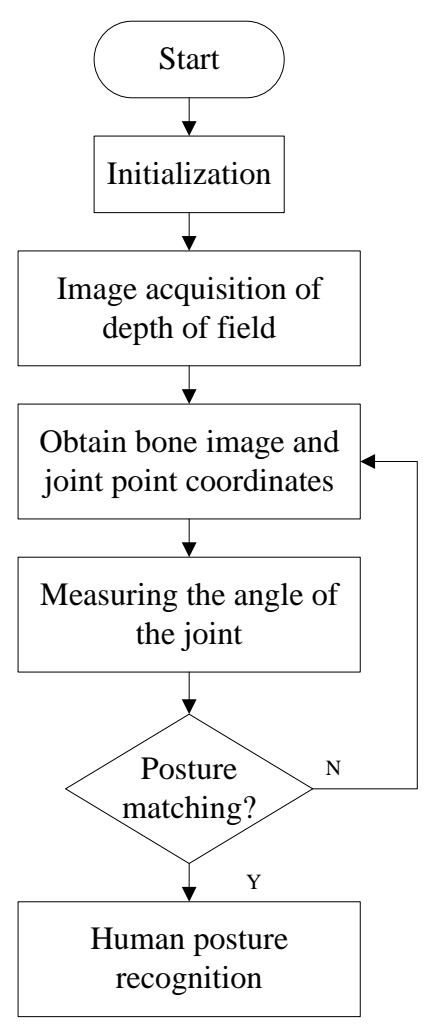

Figure 2: Flow chart of human posture recognition

\section{SYSTEM SOFTWARE DESIGN}

\subsection{Human posture recognition}

Human posture recognition mainly obtains the image acquisition of depth of field by Kinect 3D somatosensory camera. Then the human bone joint points can be identified and the spatial coordinates of bone joint points can be extracted $[7,8]$. Calculating the distance and angle between joint points by space vector, the calculation results are compared with the predefined human posture database, and the posture movement of the human body is converted to control command, transmitted by Bluetooth to self balance vehicle. The flow chart of human posture recognition is as shown in Figure 2.

\subsection{Human posture recognition}

The structure of human posture is the joints of the body, and each joint has different directions and angles in the activity. Obtaining the position information of joints and calculating the angles of joint activities is to determine human posture information. The zero point of the $x$ axis and the $y$ axis of the space coordinate system used by Kinect is the same as that of the traditional space coordinate system. The zero point of the $z$ axis is the Kinect camera and the positive direction is the positive direction of the Kinect. The two coordinate points in the coordinate system are $P_{1}\left(x_{1}, y_{1}, z_{1}\right)$ and $P_{2}\left(x_{2}, y_{2}, z_{2}\right)$. In the coordinate system, the vectors formed by two non-coincidence points $\mathrm{x}$ and point $\mathrm{y}$ are

$$
\overrightarrow{P_{1} P_{2}}=\left(x_{2}-x_{1}, y_{2}-y_{1}, z_{2}-z_{1}\right)
$$

If the third point $P_{3}\left(x_{3}, y_{3}, z_{3}\right)$ is not in the straight line $P_{1} P_{2}$, the angle formed by $P_{1} P_{2} P_{3}$ can be expressed as

$$
\angle P_{1} P_{2} P_{3} \quad \arccos \frac{\overrightarrow{P_{2} P_{1}} \overrightarrow{P_{2} P_{3}}}{\left|\overrightarrow{P_{2} P_{1}}\right|\left|\overrightarrow{P_{2} P_{3}}\right|}
$$

Therefore, the angle calculation of the acquired joint can be transformed into the calculation of the vector angle. The image of the body bone joint is shown in Figure 3. Because the calculation method of the joint angles of the left upper limb and the right upper limb is the same, taking the left side as an example, the angle of each joint of the left upper limb are calculated as follows.

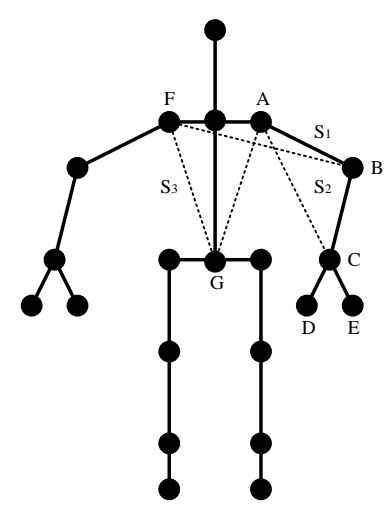

Figure 3: Image of human bone joint

\subsubsection{Palm, fist, Vhand}

The Kinect camera can only collect the information of the bone and can not be accurate to the finger state. Therefore, the state of palm and fist is calculated by the distance between the finger point $\mathrm{D}$ and the palm point $\mathrm{C}$. In addition, the judgment of $\mathrm{V}$ hand is calculated by $\angle E C D$, and the calculation process is as follows.

$$
\begin{aligned}
& |\overrightarrow{C D}|=\sqrt{\left(C_{x}-D_{x}\right)^{2}+\left(C_{y}-D_{y}\right)^{2}+\left(C_{z}-D_{z}\right)^{2}} \\
& \angle E C D \quad \arccos \frac{\overrightarrow{C E} \overrightarrow{C D}}{|\overrightarrow{C E}||\overrightarrow{C D}|}
\end{aligned}
$$

\subsubsection{Arm stretch and arm extension $\left(90^{\circ}\right)$}

The arm stretch, and arm extension were judged by calculating the angle of the left elbow. The vector of the two sides of the left elbow joint point is constructed, and the angle of the left elbow joint is calculated as follows.

$$
\angle A B C \quad \arccos \frac{\overrightarrow{B A} \overrightarrow{B C}}{|\overrightarrow{B A}||\overrightarrow{B C}|}
$$

\subsubsection{The rotation of the elbow around the upper arm}

The angle generated by the rotation of the left elbow on the upper arm can be regarded as the angle between the two intersecting planes $F A B$ and $A B C$, that is, the angle between the plane $S_{1}$ and $S_{2}$ in the Figure 3. The normal vector formula for the plane of two non collinear vectors is

$$
\vec{m}=\vec{a} \times \vec{b}=\operatorname{det}\left|\begin{array}{ccc}
i & j & k \\
a_{x} & a_{y} & a_{z} \\
b_{x} & b_{y} & b_{z}
\end{array}\right|
$$

Accordingly, the normal vector of the plane $F A B$ is calculated as

$$
\begin{aligned}
& \overrightarrow{M_{F A B}}=\overrightarrow{A F} \overrightarrow{A B}=\operatorname{det}\left|\begin{array}{ccc}
\vec{i} & \vec{j} & \vec{k} \\
A F_{x} & A F_{y} & A F_{z} \\
A B_{x} & A B_{y} & A B_{z}
\end{array}\right| \\
& \left(A F_{y} A B_{z}-\overrightarrow{A F_{z}} A B_{y}\right) i- \\
= & \left(A F_{x} A B_{z}-A F_{z} A B_{x}\right) \vec{j}+ \\
& \left(A F_{x} A B_{y}-A F_{y} A B_{x}\right) \vec{k}
\end{aligned}
$$

The normal vector of the plane $A B C$ is

$$
\overrightarrow{M_{A B C}}=\overrightarrow{B A} \overrightarrow{B C}=\operatorname{det}\left|\begin{array}{ccc}
\vec{i} & \vec{j} & \vec{k} \\
B A_{x} & B A_{y} & B A_{z} \\
B C_{x} & B C_{y} & B C_{z}
\end{array}\right|
$$




$$
\begin{aligned}
& \left(B A_{y} B C_{z}-B A_{z} B C_{y}\right) \vec{i}- \\
= & \left(B A_{x} B C_{z}-B A_{z} B C_{x}\right) \vec{j}+ \\
& \left(B A_{x} B C_{y}-B A_{y} B C_{x}\right) \vec{k}
\end{aligned}
$$

Therefore, the rotation angle of the left elbow joint can be expressed as

$$
\theta_{S_{1} S_{2}}=\arccos \frac{\overrightarrow{M_{F A B}}}{\left|\overrightarrow{M_{F A B}}\right|\left|\overrightarrow{M_{A B C}}\right|}
$$

\subsubsection{The left shoulder angle}

The angle of the left shoulder joint is the angle between the plane of the upper arm and the shoulders and the plane of the shoulders and the spine. It can be regarded as the angle between planes $F A B$ and $G F A$, that is, the angle between plane $S_{1}$ and $S_{3}$ in the Figure 3. Accordingly, the normal vector of the plane $F A B$ is calculated as above. The normal vector of the plane $G F A$ is

$$
\begin{aligned}
\overrightarrow{M_{G F A}}=\overrightarrow{F A} \overrightarrow{F G}=\operatorname{det}\left|\begin{array}{ccc}
\vec{i} & \vec{j} & \vec{k} \\
F A_{x} & F A_{y} & F A_{z} \\
F G_{x} & F G_{y} & F G_{z}
\end{array}\right| \\
\quad\left(F A_{y} F G_{z}-F A_{z} F G_{y}\right) \vec{i}- \\
=\left(F A_{x} F G_{z}-F A_{z} F G_{x}\right) \vec{j}+ \\
\quad\left(F A_{x} F G_{y}-F A_{y} F G_{x}\right) \vec{k}
\end{aligned}
$$

Therefore, the formula for calculating the angle of the left shoulder joint is

$$
\theta_{S_{1} S_{3}}=\arccos \frac{\overrightarrow{M_{B A F}} \overline{M_{G F A}}}{\left|\overrightarrow{M_{B A F}}\right|\left|\overrightarrow{M_{G F A}}\right|}
$$

\section{SYSTEM DEBUG}

From the perspective of structure, it can be divided into head, limbs and trunk. In order to control the self balancing vehicle, the human body posture is reasonably planned and defined first. The definition of action should

\begin{tabular}{|c|c|c|c|c|}
\hline Action & Definition & $\begin{array}{l}\text { Test } \\
\text { times }\end{array}$ & $\begin{array}{l}\text { Correct } \\
\text { recognition } \\
\text { times }\end{array}$ & $\begin{array}{l}\text { Recognition } \\
\text { rate }\end{array}$ \\
\hline $\begin{array}{l}\text { Arm stretch } \\
\text {,fist }\end{array}$ & advancing & 50 & 49 & $98 \%$ \\
\hline $\begin{array}{l}\text { Arm stretch, } \\
\text { palm }\end{array}$ & stop & 50 & 50 & $100 \%$ \\
\hline $\begin{array}{l}\text { Arm } \\
\text { extension, } \\
\text { V hand }\end{array}$ & retreating & 50 & 48 & $96 \%$ \\
\hline $\begin{array}{l}\text { Left turn } \\
\text { posture }\end{array}$ & turning left & 50 & 46 & $92 \%$ \\
\hline $\begin{array}{l}\text { Right turn } \\
\text { posture }\end{array}$ & $\begin{array}{l}\text { turning } \\
\text { right }\end{array}$ & 50 & 47 & $94 \%$ \\
\hline
\end{tabular}
conform to the principles of clarity, habituation and maneuverability. According to the design requirements, five kinds of postures are defined to control the vehicle, including advancing, retreating, stop, turning left and turning right. Specific action definitions are shown in Table 1. Among results, the right steering action is used to control the vehicle to turn right. The image of the right turn posture and the physical picture of the self balancing vehicle are shown in Figure 4.

Table 1: Action definition and recognition results

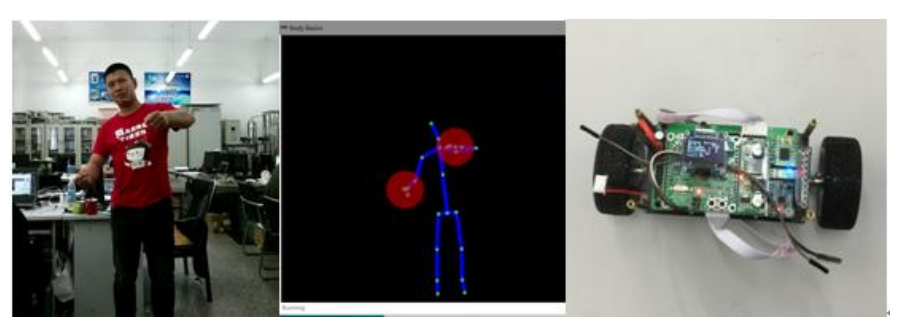

Figure 4: Right turn posture and the physical picture of the self balancing vehicle

\section{CONCLUSION}

In this paper, a self balancing vehicle control system base on human posture recognition is designed, which is implemented on Microsoft Kincet camera and Visual Studio 2012 Kinect platform. The image captured by the Kinect camera is the skeleton information of the human body, and the result of action recognition is not affected by the shape of the human body. Therefore, people with different sex and body sizes were invited to test, and the result of action recognition was almost the same. The test results show that the correct recognition rate of the action is up to $96 \%$ in the range of effective recognition distance of $1 \mathrm{~m}-5 \mathrm{~m}$, and the self balancing vehicle can achieve the corresponding action of advancing, retreating, stop, turning left and turning right. Therefore, the human posture recognition by Kinect camera can effectively control two wheeled self balancing vehicles with good controllability. The research can meet the needs of human-machine interaction control in complex environment, and it has practical significance for robot exploration, scientific research and rescue in the unmanned environment.

\section{REFERENCES}

[1] Shin, J.H., Kim, J.H., Hong, K.S. 2005. Implementation and performance evaluation of glove-based HCI methods: gesture recognition systems using fuzzy algorithm and neural network for the wearable PC. Fuzzy Logic and Applications.

[2] Wu, G.B., Li, B., Yan, J.Z. 2013. Practice of human-computer interaction with Kinect.Beijing: Posts and Telecommunications Press.

[3] Lee, H.K., Kim, J.H. 1999. An HMM-based threshold model approach for gesture recognition. Transactions on Pattern Analysis and Machine Intelligence, 21, 961-973.

[4] Han, Z., Liu, H.P., Huang, B.W. 2013. Target grasping of manipulator based on Kinect. Transactions on Intelligent Systems, 8, 149-155.

[5] Jia, P., Hu, H., Lu, T. 2007. Head gesture recognition for hands-free control of an intelligent wheelchair. Industrial Robot, 34, 60-68.

[6] Xu, T. 2012. Kinect application development practice: dialogue with machines in natural way. Machinery Industry Press.

[7] Huang, J.D. 2012. Research and implementation of dynamic gesture recognition. Wuhan: Huazhong University of Science and Technology.

[8] Weise, T., Wismer, T., Leibe, B. 2011. Online loop closure for real-time interactive 3D scanning. Computer Vision and Image Understanding, 115, 635-648.

\section{ABOUT THE AUTHORS}

Li Jiayi (1986-): Female, master's degree, graduated from Jilin University in 2011, lecturer of Jilin Institute of Chemical Technology, mainly researching on control engineering, control theory and control system simulation. 